\title{
Co-occurrence and Metabolic Biomarkers of Sensory and Motor Subtypes of Peripheral Neuropathy from Paclitaxel
}

\section{Ciao-Sin Chen}

University of Michigan

\section{Ellen M Lavoie Smith}

University of Alabama at Birmingham

\section{Kathleen A Stringer}

University of Michigan

\section{N. Lynn Henry}

University of Michigan Rogel Cancer Center

Daniel L Hertz ( $\square$ DLHertz@med.umich.edu )

University of Michigan https://orcid.org/0000-0003-0501-1035

\section{Research Article}

Keywords: chemotherapy-induced peripheral neuropathy, motor, sensory, clustering, metabolomics, predictive biomarkers

Posted Date: February 11th, 2022

DOI: https://doi.org/10.21203/rs.3.rs-1251386/v1

License: (c) (1) This work is licensed under a Creative Commons Attribution 4.0 International License. Read Full License 


\section{Abstract}

Purpose: Chemotherapy-induced peripheral neuropathy (CIPN) is the major treatment-limiting toxicity of paclitaxel, which predominantly presents as sensory symptoms, with motor symptoms in some patients. Differentiating CIPN into subtypes has been recommended to direct CIPN research. The objective of this study was to investigate whether sensory and motor CIPN are distinct subtypes with different predictive biomarkers in patients with breast cancer receiving paclitaxel.

Methods: European Organisation for Research and Treatment of Cancer Quality of Life questionnaire CIPN20 was used to evaluate CIPN. Clusters of the time course of sensory $\left(C^{2} N_{S}\right)$, motor $\left(C_{P} N_{M}\right)$, and the difference between sensory and motor $\left(\mathrm{CIPN}_{S}-\mathrm{CIPN}_{\mathrm{M}}\right)$ were identified using k-means clustering on principal component scores. Predictive metabolomic biomarkers of maximum $\mathrm{CIPN}_{S}$ and CIPN $_{M}$ were investigated using linear regressions adjusted for baseline CIPN, systemic paclitaxel exposure, and body mass index.

Results: More sensory than motor CIPN was found (CIPN ${ }_{S}$ change: mean=10.8, ranged [-3.3, 52.1]; $C$ IPN $N_{M}$ change: mean=3.5, range: $[-7.5,35.0])$. Three groups were identified with No CIPN, Mixed CIPN, and Sensory-dominant CIPN (maximum CIPN $_{S}$ : mean=12.7 vs. 40.9 vs. $74.3, p<0.001$; maximum CIPN $_{\mathrm{M}}$ : mean $=5.4$ vs. 25.5 vs. 36.1 , $p<0.001$; average $\mathrm{CIPN}_{S}-\mathrm{CIPN}_{\mathrm{M}}$ : mean=2.8 vs. 5.8 vs. $24.9, p<0.001$ ). Biomarkers of motor CIPN were similar to previously identified biomarkers of sensory CIPN, including lower serum histidine $(p=0.029)$.

Conclusion: Our findings suggest that sensory and motor CIPN co-occur and may not have differentiating metabolic biomarkers. These findings need to be validated in larger cohorts of patients treated with paclitaxel and other neurotoxic agents to determine the optimal approach to predict, prevent, and treat CIPN and improve patients' outcomes.

\section{Introduction}

Chemotherapy-induced peripheral neuropathy (CIPN) is a debilitating side effect caused by several commonly used chemotherapy agents, including taxanes, platinums, and vinca alkaloids. CIPN causes sensory and motor symptoms in the upper and lower extremities that can progress to loss of function. CIPN symptoms can persist for years after chemotherapy [1-5] and severely diminish patient's quality of life $[3,6]$. There are no effective agents to prevent or treat CIPN, so the only recommended management is to reduce or delay chemotherapy dosing or discontinue treatment, which reduces treatment efficacy and patient survival [7].

The clinical presentation of CIPN differs between and within drug classes [8,9]. Paclitaxel-induced peripheral neuropathy presents with predominantly sensory symptoms including tingling, numbness, or burning pain $[8,9]$. Some paclitaxel-treated patients also experience changes in motor functions caused 
by muscle weakness [8-11], which is particularly concerning to older patients with cancer because they can lead to increased risk of falls $[12,13]$.

Expert panels have suggested differentiating CIPN into its sensory and motor subtypes in order to investigate pathophysiological mechanisms and develop preventive and treatment interventions $[14,15]$. Additionally, if sensory and motor CIPN have distinct mechanisms, it would be reasonable to expect that they have distinct predictive biomarkers, which could help identify which patients should be prioritized for prevention trials or monitored more closely during treatment $[14,16,17]$. A previous analysis of patientreported CIPN data prospectively collected from a cohort of patients receiving various neurotoxic chemotherapy agents identified four CIPN symptom subtype clusters: Sensory, Motor-dominant Mixed, Sensory-dominant Mixed, and a less clear autonomic cluster [18]. The prior study did not include a sufficient number of patients receiving individual neurotoxic agents to confirm this clustering pattern within each drug. Additionally, almost all biomarker studies have combined sensory and motor CIPN into a single endpoint or conducted analyses of sensory CIPN [19-28]; few studies have investigated predictive biomarkers of motor CIPN subtype [29].

The objective of this study was to investigate whether sensory and motor CIPN are distinct subtypes with different predictive metabolic biomarkers in patients with breast cancer receiving weekly paclitaxel treatment. We conducted an exploratory analysis of CIPN data collected weekly throughout treatment via a patient-reported outcome (PRO) questionnaire to evaluate the time course of sensory and motor CIPN, determine how sensory and motor CIPN co-occurred, and attempt to identify predictive metabolic biomarkers of the motor CIPN subtype.

\section{Methods}

\section{Study Patients}

This study is an exploratory analysis of a previously described cohort (NCT02338115) [19]. Adult female patients with stage I-III or oligometastatic breast cancer scheduled to receive paclitaxel $80 \mathrm{mg} / \mathrm{m}^{2} 1$-hour infusion weekly for 12 doses were eligible to enroll in this observational study. Patients were excluded if they had prior or concurrent neurotoxic chemotherapy (taxane, vinca alkaloid, platinum, bortezomib, or thalidomide), concurrent duloxetine treatment or enrollment in a clinical study of any neuroprotective agent, existing severe peripheral neuropathy affecting activities of daily living, or known family history of hereditary peripheral neuropathy or Charcot-Marie-Tooth disease. Patients who withdrew from the study or discontinued paclitaxel treatment before receiving at least 3 doses were excluded from the analysis. Demographic and lifestyle information was collected from patients at baseline. All instances of treatment disruption including dose delay, dose decrease, and treatment discontinuation were determined from the electronic medical record. All patients signed written informed consent, and the study was approved by Institutional Review Boards of the University of Michigan Medical School (IRBMED) (HUM00086253). Baseline metabolomics [27] and nutrients [28] were measured in a subset of patients who consented to additional biomarker analyses. 


\section{Patient-reported CIPN}

Patient-reported CIPN was evaluated at baseline and weekly throughout paclitaxel treatment using European Organisation for Research and Treatment of Cancer (EORTC) Quality of Life Questionnaire Chemotherapy-Induced Peripheral Neuropathy (CIPN20). The 20 questions ask about 9 sensory, 8 motor, and 3 autonomic symptoms, which are graded on a scale from 1 to 4 ( $1=$ not at all, $2=a$ little, $3=$ =quite a bit, and 4=very much). Sensory CIPN (CIPN $)$ was defined as the sum score of the first 8 sensory items (excluding the ototoxicity item), which are the most common sensory symptoms, including tingling, numbness, or pain in the hands and feet, and having problems standing or walking due to difficulty feeling the ground, and having difficulty distinguishing between hot and cold water [19]. Motor CIPN $\left(\mathrm{CIPN}_{\mathrm{M}}\right)$ was defined as the 8 motor items, including cramps in the hands and feet, and having problems holding a pen to write, having difficulty opening a jar or bottle due to weakness in the hands, having difficulty walking or climbing stairs due to weakness in the legs, and having difficulty manipulating small objects or using the pedals. All scores were converted to a scale of 0-100 with higher scores indicating more severe CIPN [30].

\section{Statistical Analysis}

\section{CIPN Subtypes and Clustering Analysis}

The time course of sensory $\left(\mathrm{CIPN}_{S}\right)$, motor $\left(\mathrm{CIPN}_{\mathrm{M}}\right)$, the difference between sensory and motor CIPN $\left(\mathrm{CIPN}_{\mathrm{S}}-\mathrm{CIPN}_{\mathrm{M}}\right)$, and $\mathrm{CIPN}_{\mathrm{S}}$ vs. CIPN $\mathrm{M}_{\mathrm{M}}$ were plotted for all patients throughout paclitaxel treatment for visual inspection using R 3.6.3. Clusters of the time course of $C I P N_{S}$, CIPN $N_{M}$, and $C_{P} N_{S}-C_{P} N_{M}$ were identified using k-means clustering on principal component analysis (PCA) scores. CIPN scores at time points with $\geq 80 \%$ valid data were included, and CIPN scores at missing time points were imputed using interpolation and extrapolation from individual patient's time course. $\mathrm{CIPN}_{S}$ and $\mathrm{CIPN}_{\mathrm{M}}$ were scaled to 0-1, and CIPN $_{S}-$ CIPN $_{M}$ were scaled to (-1)-1. The number of clusters was determined by the elbow method of the within-cluster sum of squares. A thousand sets of random centers were tested, and the centers with lowest within-cluster sum of squares and highest between-cluster sum of squares were selected. To depict the characteristics of each cluster, CIPN scores, treatment administration information (paclitaxel systemic exposure and treatment disruptions), and demographics were compared between clusters using analysis of variance (ANOVA) for continuous variables and Chi-square tests for binary variables with $a=$ 0.05. If significant, post hoc analyses were performed using pairwise comparisons with Bonferroni correction.

\section{Metabolic Biomarker Analysis}

Baseline metabolomics [27] and nutrients [28] data were used in metabolic biomarker analyses. Missing data of metabolomics were imputed with half of the limit of detection. All measurements were $\log _{2}$ transformed and z-score normalized. Linear regression analyses were used to identify biomarkers associated with maximum $\mathrm{CIPN}_{S}$ and $\mathrm{CIPN}_{\mathrm{M}}$ after adjusting for baseline $\mathrm{CIPN}_{\mathrm{S}}$ and $\mathrm{CIPN}_{\mathrm{M}}$, paclitaxel 
maximum concentration $\left(\mathrm{C}_{\max }\right)$, and time above paclitaxel concentration threshold $\left(\mathrm{T}_{\mathrm{C}>0.05}\right)$, and two-way interaction terms were included if significant [19]. Baseline clinical covariates such as prior or concurrent chemotherapy, age, race, body mass index, pretreatment neuropathy, diabetes, and use of pain medication were also included if significant.

\section{Results}

\section{Patient-reported CIPN}

A total of 60 patients were enrolled, but one patient who discontinued treatment after 2 doses due to nonneuropathy toxicity was excluded from the analysis. The average age of the 59 patients included in the analyses was 52 and $>90 \%$ were Caucasian (Table 1). Most patients had no or limited sensory or motor CIPN at baseline (CIPN $N_{S}$ : mean=1.3 (range: $\left.[0,12.5]\right)$, $C I P N_{M}$ : mean=2.7 (range: $\left.[0,25.0]\right)$ ). As expected, sensory and motor CIPN increased throughout paclitaxel treatment, and the increases were larger for sensory than motor $\operatorname{CIPN}\left(\mathrm{CIPN}_{S}\right.$ average change throughout treatment: mean=10.8 (range: [-3.3, 52.1]); $\mathrm{CIPN}_{\mathrm{M}}$ average change throughout treatment: mean=3.5 (range: $\left.[-7.5,35.0]\right)$ ) (Figure 1). 
Table 1

Demographics of 59 patients that were included in the analysis.

\begin{tabular}{|c|c|c|}
\hline Variable & Definition & $\begin{array}{l}\mathbf{N}(\%) \text { or } \\
\text { mean } \\
\text { [range] }\end{array}$ \\
\hline Age & Age at enrollment (years) & $52.3[28,71]$ \\
\hline Race & Caucasian & $54(91.5 \%)$ \\
\hline Body mass index & $\mathrm{kg} / \mathrm{m}^{2}$ & $\begin{array}{l}28.3[19.2, \\
45.8]\end{array}$ \\
\hline \multirow[t]{2}{*}{ Other cancer treatment } & Prior AC & $55(93.2 \%)$ \\
\hline & Concurrent H/P & $29(49.2 \%)$ \\
\hline \multirow[t]{5}{*}{ Paclitaxel treatment } & $\begin{array}{l}\text { Cumulative dose received } \\
\left(\mathrm{mg} / \mathrm{m}^{2}\right)\end{array}$ & $\begin{array}{l}885[432 \\
986]\end{array}$ \\
\hline & Number of doses received & $11.2[6,12]$ \\
\hline & Relative dose intensity & $\begin{array}{l}0.887 \\
{[0.379,1]}\end{array}$ \\
\hline & Treatment disruption ${ }^{a}$ & $35(59.3 \%)$ \\
\hline & $\begin{array}{l}\text { Number of doses at first } \\
\text { disruption }\end{array}$ & $9[2,12]$ \\
\hline \multirow[t]{2}{*}{ Paclitaxel systemic exposure } & $\mathrm{C}_{\max }(\mathrm{ng} / \mathrm{mL})(\mathrm{N}=56)$ & $\begin{array}{l}2375[907 \\
4340]\end{array}$ \\
\hline & $\mathrm{T}_{\mathrm{C}>0.05}(\mathrm{hr})(\mathrm{N}=58)$ & $10.7[7,22]$ \\
\hline \multirow[t]{4}{*}{ Baseline neuropathy predictors } & Diabetes or $\mathrm{Hb}_{\mathrm{A} 1 \mathrm{C}} \geq 6.5 \%(\mathrm{~N}=52)$ & $13(25 \%)$ \\
\hline & Use of alcohol $(\mathrm{N}=58)$ & $27(46.6 \%)$ \\
\hline & Use of pain medication & $7(11.9 \%)$ \\
\hline & $\begin{array}{l}\text { Vitamin D insufficiency } \\
(\leq 20 \mathrm{ng} / \mathrm{mL})(\mathrm{N}=36)\end{array}$ & $17(47.2 \%)$ \\
\hline \multirow[t]{2}{*}{ Sensory CIPN $\left(\mathrm{CIPN}_{S}\right)$} & Baseline & $1.3[0,12.5]$ \\
\hline & Maximum during treatment & $26.4[0,87.5]$ \\
\hline Motor CIPN $\left(\mathrm{CIPN}_{\mathrm{M}}\right)$ & Baseline & $2.7[0,25]$ \\
\hline
\end{tabular}

a All-cause (CIPN- and non-CIPN-related) treatment disruption includes any dose delay, dose decrease, or early treatment discontinuation.

AC: Adriamycin (doxorubicin) and cyclophosphamide. H/P: trastuzumab or pertuzumab 


\begin{tabular}{|c|c|c|}
\hline \multirow[t]{2}{*}{ Variable } & Definition & $\begin{array}{l}\mathbf{N}(\%) \text { or } \\
\text { mean } \\
\text { [range] }\end{array}$ \\
\hline & Maximum during treatment & $13.8[0,58.3]$ \\
\hline \multirow[t]{3}{*}{$\begin{array}{l}\text { Difference between sensory and motor CIPN } \\
\left(\text { CIPN }_{S}-\text { CIPN }_{M}\right)\end{array}$} & Baseline & $\begin{array}{l}-2[-19.4 \\
12.5]\end{array}$ \\
\hline & Minimum during treatment & $\begin{array}{l}-3.4[-29.2, \\
8.3]\end{array}$ \\
\hline & Maximum during treatment & $\begin{array}{l}17.7[-5.6, \\
61.1]\end{array}$ \\
\hline \multicolumn{3}{|c|}{$\begin{array}{l}\text { a All-cause (CIPN- and non-CIPN-related) treatment disruption includes any dose delay, dose decrease, } \\
\text { or early treatment discontinuation. }\end{array}$} \\
\hline \multicolumn{3}{|c|}{ AC: Adriamycin (doxorubicin) and cyclophosphamide. H/P: trastuzumab or pertuzumab } \\
\hline
\end{tabular}

The time course of the difference between sensory and motor CIPN (CIPN $\left.-\mathrm{CIPN}_{M}\right)$ indicates that most patients had greater sensory symptoms $\left(\mathrm{CIPN}_{S}-\mathrm{CIPN}_{\mathrm{M}}>0\right)$ or similar sensory and motor symptoms $\left(\mathrm{CIPN}_{\mathrm{S}}-\mathrm{CIPN}_{\mathrm{M}} \approx 0\right)$; only one patient experienced meaningfully greater motor than sensory symptoms (Figure 2). Patients who had similar sensory and motor CIPN was comprised of two groups, those who experienced no or limited symptoms of either, and those who experienced both symptoms with approximately equal severity.

\section{Clustering of CIPN Subtypes}

The time course of sensory CIPN, motor CIPN, and the difference between the two $\left(\mathrm{CIPN}_{S}-\mathrm{CIPN}_{M}\right)$ from baseline to week 11 were included in k-means clustering using PCA scores. Three distinctive clusters were identified, which were annotated as $\operatorname{No} \operatorname{CIPN}(\mathrm{N}=37$, black), Mixed $\operatorname{CIPN}(\mathrm{N}=16$, red), and Sensorydominant $\operatorname{CIPN}(\mathrm{N}=6$, green) based on the CIPN score profile (Figures 3 and S1). These annotations are further supported by the differences in CIPN symptom subtype severity (Table 2 and S1). No CIPN had minimal sensory or motor CIPN (maximum $\mathrm{CIPN}_{S}$ during treatment: mean=12.7, maximum $\mathrm{CIPN}_{\mathrm{M}}$ during treatment: mean=5.4) compared to Mixed $\operatorname{CIPN}\left(\mathrm{CIPN}_{S}=40.9, \mathrm{CIPN}_{\mathrm{M}}=25.5\right.$, both $\left.\mathrm{p}<0.001\right)$. Sensorydominant CIPN also had both sensory and motor CIPN, but only sensory CIPN was greater than the Mixed CIPN group ( $\left.C_{I P N}=74.3, p<0.001\right)$. Sensory-dominant $C I P N$ had higher CIPN $_{S}-C_{I P N_{M}}$ than Mixed CIPN, further indicating that the primary distinction between these two groups is the dominance of sensory symptoms (average $\mathrm{CIPN}_{S}-\mathrm{CIPN}_{\mathrm{M}}$ during treatment: mean=24.9 vs. $5.8, p<0.001$ ). 
Table 2

Patient-reported CIPN scores and treatment disruption between clusters. ${ }^{\text {a }}$

\begin{tabular}{|c|c|c|c|c|c|c|}
\hline Variable & Definition & $\begin{array}{l}\text { No } \\
\text { CIPN } \\
(\mathrm{N}=37)\end{array}$ & $\begin{array}{l}\text { Mixed } \\
\text { CIPN } \\
(\mathrm{N}=16)\end{array}$ & $\begin{array}{l}\text { Sensory- } \\
\text { dominant CIPN } \\
(\mathrm{N}=6)\end{array}$ & $\begin{array}{l}\text { p- } \\
\text { value }^{b}\end{array}$ & $\begin{array}{l}\text { Pairwise } \\
\text { Tests }^{c}\end{array}$ \\
\hline $\mathrm{CIPN}_{S}$ & $\begin{array}{l}\text { Maximum during } \\
\text { treatment }\end{array}$ & $\begin{array}{l}12.7 \\
(8.4)\end{array}$ & $\begin{array}{l}40.9 \\
(10.7)\end{array}$ & $74.3(12.8)$ & $<0.001$ & $3>2>1$ \\
\hline $\mathrm{CIPN}_{\mathrm{M}}$ & $\begin{array}{l}\text { Maximum during } \\
\text { treatment }\end{array}$ & $\begin{array}{l}5.4 \\
(5.1)\end{array}$ & $\begin{array}{l}25.5 \\
(14.2)\end{array}$ & $36.1(20)$ & $<0.001$ & $3=2>1$ \\
\hline $\mathrm{CIPN}_{S}-\mathrm{CIPN}_{\mathrm{M}}$ & $\begin{array}{l}\text { Average during } \\
\text { treatment }\end{array}$ & $\begin{array}{l}2.8 \\
(4.7)\end{array}$ & $\begin{array}{l}5.8 \\
(8.4)\end{array}$ & $24.9(6.7)$ & $<0.001$ & $3>2=1$ \\
\hline \multirow[t]{2}{*}{$\begin{array}{l}\text { Paclitaxel } \\
\text { administration }\end{array}$} & $\begin{array}{l}\text { Relative dose } \\
\text { intensity }\end{array}$ & $\begin{array}{l}0.94 \\
(0.10)\end{array}$ & $\begin{array}{l}0.78 \\
(0.18)\end{array}$ & $0.85(0.12)$ & $<0.001$ & $1>2$ \\
\hline & $\begin{array}{l}\text { Early } \\
\text { discontinuation }\end{array}$ & $10.8 \%$ & $62.5 \%$ & $66.7 \%$ & $<0.001$ & $3=2>1$ \\
\hline $\begin{array}{l}\text { Paclitaxel } \\
\text { systemic exposure }\end{array}$ & $\mathrm{T}_{\mathrm{C}>0.05}(\mathrm{hr})$ & $\begin{array}{l}9.9 \\
(1.7)\end{array}$ & $12(4)$ & $11.8(1.7)$ & 0.019 & \\
\hline \multicolumn{7}{|c|}{$\begin{array}{l}\text { a Continuous variables are shown as mean (standard deviation), binary variables are shown as } \\
\text { percentage. }\end{array}$} \\
\hline \multicolumn{7}{|c|}{${ }^{b} p$-values that are smaller than 0.001 are shown as $p<0.001$. } \\
\hline
\end{tabular}

We then explored whether the differences between the clusters were associated with paclitaxel administration or paclitaxel systemic exposure. Patients in the No CIPN group had higher paclitaxel dose intensity than the Mixed $\operatorname{CIPN}(0.94$ vs. $0.78, \mathrm{p}=0.003)$, and fewer early treatment discontinuations than the Mixed or Sensory-Dominant CIPN groups ( $10.8 \%$ vs. $62.5 \%$ or $66.7 \%, p=0.007$ or $p<0.001$, respectively). There were no differences in dose intensity or treatment disruption between the Mixed and Sensory-Dominant CIPN groups $(\mathrm{p}=0.261$ and $\mathrm{p}=0.910)$. Interestingly, both Mixed and Sensory-dominant CIPN had nominally higher paclitaxel systemic exposure than No $C I P N\left(T_{C>0.05}=12.0\right.$ and 11.8 vs 9.9 hours), but pairwise comparisons were not significant (Table 2 and S1).

\section{Metabolic Biomarkers of CIPN Subtypes}

Prior analyses in this dataset found that lower levels of histidine, phenylalanine, threonine, and vitamin D were associated with more severe sensory $\operatorname{CIPN}[27,28]$ and remained significant after adjusting for relevant covariates including baseline CIPN severity, paclitaxel systemic exposure, and body mass index (Table 3). In the current analysis, lower histidine was also associated with more severe motor CIPN (Table 
3 and Figure 4). The other biomarkers that had inverse associations with sensory CIPN had similar, though weaker and not statistically significant, associations with motor CIPN (Table 3 and Figure S2). None of the other tested metabolomic biomarkers were associated with sensory or motor CIPN (Table S2).

Table 3

Metabolomics and nutrient biomarkers of sensory and motor CIPN.

\begin{tabular}{|c|c|c|c|c|}
\hline & \multicolumn{2}{|c|}{ Sensory CIPN } & \multicolumn{2}{|c|}{ Motor CIPN } \\
\hline & $r$ & p-value ${ }^{1}$ & $\mathrm{r}$ & $p$-value ${ }^{a}$ \\
\hline Histidine & -0.376 & 0.006 & -0.254 & 0.029 \\
\hline Phenylalanine & -0.336 & 0.011 & -0.124 & 0.110 \\
\hline Threonine & -0.283 & 0.014 & -0.057 & 0.400 \\
\hline Vitamin D concentration & -0.352 & 0.043 & -0.136 & 0.106 \\
\hline \multicolumn{5}{|c|}{$\begin{array}{l}\text { a p-values from linear regression adjusted for baseline CIPN, paclitaxel systemic exposure, cumulative } \\
\text { dose, relative dose intensity, and body mass index. }\end{array}$} \\
\hline r: Correlation coefficient & & & & \\
\hline
\end{tabular}

\section{Discussion}

The major treatment-limiting toxicity of paclitaxel is CIPN [31-33], which predominantly presents as sensory symptoms [8, 9], with motor symptoms in some patients [8-11]. There are no known effective treatments that can prevent or ameliorate CIPN [7], which can be due to the unclear mechanism of CIPN [31]. Differentiation of CIPN into symptom subtypes has been recommended to improve the success of CIPN intervention and biomarker discovery trials $[14,16,17]$. This exploratory analysis used PRO CIPN data collected prospectively during weekly paclitaxel treatment to investigate whether sensory and motor CIPN are distinct subtypes. As expected, more sensory than motor CIPN was reported. Interestingly, patient groups were identified with no CIPN, mixed CIPN, and sensory-dominant CIPN, but not motordominant CIPN. The metabolomics and nutrient biomarker results for motor CIPN were similar to those previously reported for sensory CIPN, suggesting there may not be distinct predictive metabolic biomarkers for motor CIPN from paclitaxel treatment.

Patients with breast cancer receiving taxane treatment experience both sensory and motor CIPN [34], but sensory CIPN is more common, especially with paclitaxel [6, 35-37]. Our findings are consistent with previous studies that sensory symptoms are more common, and we found that when motor symptoms occur, they usually co-occur with sensory symptoms. We did not find an appreciable group of patients with motor-only or motor-dominant CIPN; the only patient who experienced motor-dominant symptoms was also the only patient with appreciable motor CIPN symptoms at baseline (Figure 2, $\mathrm{CIPN}_{\mathrm{M}}=25$ ). A prior case report described a patient who developed objective evidence of motor-only CIPN based on 
nerve function impairment, however, the motor symptoms were not detected by a PRO questionnaire [38]. Sensitive nerve conduction studies have found that distal motor symptoms can occur alone [39], but the evidence from patient-report and clinician-assessment indicates that this is rare and motor symptoms are more likely to be a progression from sensory symptoms $[39,40]$.

Our clustering analysis of paclitaxel-treated patients identified No CIPN, Mixed CIPN, and Sensorydominant CIPN groups. A previous analysis in patients with any cancer receiving any neurotoxic chemotherapy identified four clusters: Sensory, Motor-dominant Mixed, Sensory-dominant Mixed, and a less clear Autonomic cluster; a subgroup analysis of patients receiving paclitaxel alone $(\mathrm{N}=33)$ or with the somewhat neurotoxic carboplatin $(\mathrm{N}=50)$ had similar clustering results [18]. While both studies agree that there is a lack of a motor-only subgroup from paclitaxel treatment, our study did not identify evidence of a Motor-dominant group. One of the core symptoms in their Motor-dominant Mixed cluster was difficulty manipulating small objects [18], which can be indicative of either motor or sensory impairment [40, 41]. We explored alternative definitions of the motor subtype that removed the two potentially confounded items, manipulating small objects and using pedals [41, 42], and still did not identify any motor-only or motor-dominant clusters (data not shown). The other difference between the studies is that we did not identify an autonomic cluster. We did not analyze the autonomic subscale of the CIPN20 because this subscale has been shown to be unstable and an unreliable indicator of CIPN [41-44], and autonomic CIPN symptoms are uncommon from paclitaxel treatment $[3,45]$.

Our analysis found that predictive metabolic biomarkers for motor CIPN were generally similar to those we previously reported for sensory CIPN [27, 28]; we did not find any evidence of distinct motor CIPN biomarkers. The majority of paclitaxel CIPN biomarker research has not differentiated between CIPN subtypes or has focused exclusively on the sensory subtype [19-28]. We are aware of only one study, also conducted in patients with breast cancer receiving paclitaxel, that found higher baseline levels of sphingolipids were associated with higher incidence of motor, but not sensory, CIPN [29]. These hypothesis-generating results require validation in independent patient cohorts to determine whether baseline lipid levels may be predictive biomarkers of motor CIPN in patients treated with paclitaxel and perhaps other neurotoxic chemotherapy agents.

Overall, our results cast some doubt on recent recommendations for differentiating between motor and sensory CIPN in clinical trials and biomarker research, at least for paclitaxel $[14,16,17]$. The lack of a motor-only or motor-dominant subtype, and previous evidence that motor symptoms may be a progression from sensory symptoms, instead favors focusing prevention and treatment efforts on earlier sensory symptoms to avoid onset of the clinically troubling motor effects [12, 13]. In terms of CIPN monitoring, PRO questionnaires have several advantages over clinician-assessment [30,46-49], one of which may be the ability to more clearly differentiate sensory and motor symptoms. This would be particularly helpful if someday there were treatments that were specifically effective in one or the other subtype. Finally, although it was only a single patient, our results indicate that patients with baseline motor neuropathy symptoms may have elevated risk of treatment-induced motor CIPN. This suggests that more sensitive baseline screening may help identify patients who should be considered for non-

Page $10 / 20$ 
neurotoxic alternatives or enhanced CIPN monitoring, perhaps using novel wearable or app-based monitoring strategies [50].

This analysis used sensitive PRO data collected weekly throughout treatment in a relatively homogeneous cohort of patients with breast cancer receiving paclitaxel and investigated metabolic and nutrient biomarkers of motor CIPN. Despite these strengths, this study has several limitations. First, the modest sample size limits our confidence in concluding there is no motor-dominant patient group or motor-specific biomarkers. Second, although PRO are more sensitive and reliable than CTCAE [49], there is evidence that the CIPN20 subscale structure may have suboptimal structural validity [41, 43, 44], and the subscales may not optimally characterize the symptom subtypes [42]. Third, we only examined a subset of metabolites and nutrients including vitamin $D$, vitamin $B_{12}$, folate, homocysteine, and metabolite data generated by nuclear magnetic resonance spectroscopy; this metabolomics approach is less sensitive and results in fewer named metabolites than liquid chromatography-mass spectroscopy. Our ongoing lipidomics and proteomics analyses will attempt to validate previously reported biomarker candidates of sensory and/or motor CIPN [26, 29]. Finally, our findings are likely confined to only paclitaxel, and further research is needed to determine whether they should be generalized to docetaxel and perhaps other classes of neurotoxic chemotherapy. We plan to investigate symptom subtype clusters and predictive biomarkers in SWOG 1714 (NCT03939481), a recently completed observational clinical trial investigating clinical and physiologic predictors of CIPN in patients receiving paclitaxel or docetaxel treatment.

In conclusion, our findings suggest that in paclitaxel-treated patients, introduce the possibility that sensory and motor are not independent CIPN subtypes. Rather, motor symptoms co-occur with sensory symptoms. Prediction, prevention, and treatment of CIPN from paclitaxel should focus primarily on the more common sensory subtype. These findings need to be validated in larger cohorts of patients treated with paclitaxel and then tested in cohorts receiving docetaxel and other neurotoxic agents to determine the optimal approach to evaluate interventions for CIPN prevention and treatment, which could improve clinical outcomes in patients with cancer.

\section{Declarations}

\section{Funding}

This research was funded in part by the National Center for Advancing Translation Sciences (NCATS National Cancer 2UL1TR000433, KL2TR000434) (DLH) and National Cancer Institute (NCl P30CA046592) through use of the UM Pharmacokinetics Core. Dr. Kathleen Stringer's effort was supported, in part, by a Grant from the National Institute of General Medical Sciences (NIGMS R35 GM136312). The content is solely the responsibility of the authors and does not necessarily represent the official views of the NIH, NCATS, NCl, or NIGMS. 


\section{Competing Interests}

The authors have no relevant financial or non-financial interests to disclose.

\section{Author Contributions}

All authors contributed to the study conception and design. Data analyses were performed by Ciao-Sin Chen. The first draft of the manuscript was written by Ciao-Sin Chen and Dan L Hertz, and all authors commented on previous versions of the manuscript. All authors read and approved the final manuscript.

\section{Data Availability}

The datasets analyzed during the current study are not publicly available due to patient privacy requirements but are available from the corresponding author on reasonable request.

\section{Ethics approval}

This study was performed in line with the principles of the Declaration of Helsinki. Approval was granted by Institutional Review Boards of the University of Michigan Medical School (IRBMED) (HUM00086259).

\section{Consent to participate}

Informed consent was obtained from all individual participants included in the study.

\section{References}

1. Hershman DL, Unger JM, Crew KD, Till C, Greenlee H, Minasian LM, Moinpour CM, Lew DL, Fehrenbacher L, Wade JL, 3rd et al: Two-Year Trends of Taxane-Induced Neuropathy in Women Enrolled in a Randomized Trial of Acetyl-L-Carnitine (SWOG S0715). Journal of the National Cancer Institute 2018, 110(6):669-676.

2. Mustafa Ali M, Moeller M, Rybicki L, Moore HCF: Long-term peripheral neuropathy symptoms in breast cancer survivors. Breast cancer research and treatment 2017, 166(2):519-526.

3. Simon NB, Danso MA, Alberico TA, Basch E, Bennett AV: The prevalence and pattern of chemotherapy-induced peripheral neuropathy among women with breast cancer receiving care in a large community oncology practice. Qual Life Res 2017, 26(10):2763-2772.

4. Bao T, Basal C, Seluzicki C, Li SQ, Seidman AD, Mao JJ: Long-term chemotherapy-induced peripheral neuropathy among breast cancer survivors: prevalence, risk factors, and fall risk. Breast cancer research and treatment 2016, 159(2):327-333. 
5. Osmani K, Vignes S, Aissi M, Wade F, Milani P, Lévy BI, Kubis N: Taxane-induced peripheral neuropathy has good long-term prognosis: a 1- to 13-year evaluation. Journal of neurology 2012, 259(9):1936-1943.

6. Kuroi K, Shimozuma K: Neurotoxicity of taxanes: symptoms and quality of life assessment. Breast cancer (Tokyo, Japan) 2004, 11(1):92-99.

7. Loprinzi CL, Lacchetti C, Bleeker J, Cavaletti G, Chauhan C, Hertz DL, Kelley MR, Lavino A, Lustberg MB, Paice JA et al: Prevention and Management of Chemotherapy-Induced Peripheral Neuropathy in Survivors of Adult Cancers: ASCO Guideline Update. Journal of clinical oncology : official journal of the American Society of Clinical Oncology 2020, 38(28):3325-3348.

8. Miltenburg NC, Boogerd W: Chemotherapy-induced neuropathy: A comprehensive survey. Cancer treatment reviews 2014, 40(7):872-882.

9. Quasthoff S, Hartung HP: Chemotherapy-induced peripheral neuropathy. Journal of neurology 2002, 249(1):9-17.

10. Freilich RJ, Balmaceda C, Seidman AD, Rubin M, DeAngelis LM: Motor neuropathy due to docetaxel and paclitaxel. Neurology 1996, 47(1):115-118.

11. Boyette-Davis JA, Cata JP, Driver LC, Novy DM, Bruel BM, Mooring DL, Wendelschafer-Crabb G, Kennedy WR, Dougherty PM: Persistent chemoneuropathy in patients receiving the plant alkaloids paclitaxel and vincristine. Cancer chemotherapy and pharmacology 2013, 71(3):619-626.

12. Winters-Stone KM, Horak F, Jacobs PG, Trubowitz P, Dieckmann NF, Stoyles S, Faithfull S: Falls, Functioning, and Disability Among Women With Persistent Symptoms of Chemotherapy-Induced Peripheral Neuropathy. Journal of clinical oncology : official journal of the American Society of Clinical Oncology 2017, 35(23):2604-2612.

13. Kolb NA, Smith AG, Singleton JR, Beck SL, Stoddard GJ, Brown S, Mooney K: The Association of Chemotherapy-Induced Peripheral Neuropathy Symptoms and the Risk of Falling. JAMA Neurol 2016, 73(7):860-866.

14. Dorsey SG, Kleckner IR, Barton D, Mustian K, O'Mara A, St Germain D, Cavaletti G, Danhauer SC, Hershman DL, Hohmann AG et al: The National Cancer Institute Clinical Trials Planning Meeting for Prevention and Treatment of Chemotherapy-Induced Peripheral Neuropathy. Journal of the National Cancer Institute 2019, 111(6):531-537.

15. Themistocleous AC, Crombez G, Baskozos G, Bennett DL: Using stratified medicine to understand, diagnose, and treat neuropathic pain. Pain 2018, 159 Suppl 1(Suppl 1):S31-s42.

16. Gewandter JS, Brell J, Cavaletti G, Dougherty PM, Evans S, Howie L, McDermott MP, O'Mara A, Smith AG, Dastros-Pitei D et al: Trial designs for chemotherapy-induced peripheral neuropathy prevention: ACTTION recommendations. Neurology 2018, 91(9):403-413.

17. Chan A, Hertz DL, Morales M, Adams EJ, Gordon S, Tan CJ, Staff NP, Kamath J, Oh J, Shinde S et al: Biological predictors of chemotherapy-induced peripheral neuropathy (CIPN): MASCC neurological complications working group overview. Supportive care in cancer : official journal of the Multinational Association of Supportive Care in Cancer 2019, 27(10):3729-3737. 
18. Wang M, Cheng HL, Lopez V, Sundar R, Yorke J, Molassiotis A: Redefining chemotherapy-induced peripheral neuropathy through symptom cluster analysis and patient-reported outcome data over time. BMC Cancer 2019, 19(1):1151.

19. Hertz DL, Kidwell KM, Vangipuram K, Li F, Pai MP, Burness M, Griggs JJ, Schott AF, Van Poznak C, Hayes DF et al: Paclitaxel Plasma Concentration after the First Infusion Predicts Treatment-Limiting Peripheral Neuropathy. Clinical cancer research : an official journal of the American Association for Cancer Research 2018, 24(15):3602-3610.

20. Chua KC, Xiong C, Ho C, Mushiroda T, Jiang C, Mulkey F, Lai D, Schneider BP, Rashkin SR, Witte JS et al: Genomewide Meta-Analysis Validates a Role for S1PR1 in Microtubule Targeting Agent-Induced Sensory Peripheral Neuropathy. Clinical pharmacology and therapeutics 2020, 108(3):625-634.

21. Komatsu M, Wheeler HE, Chung S, Low SK, Wing C, Delaney SM, Gorsic LK, Takahashi A, Kubo M, Kroetz DL et al: Pharmacoethnicity in Paclitaxel-Induced Sensory Peripheral Neuropathy. Clinical cancer research : an official journal of the American Association for Cancer Research 2015, 21(19):4337-4346.

22. Abraham JE, Guo Q, Dorling L, Tyrer J, Ingle S, Hardy R, Vallier A-L, Hiller L, Burns R, Jones L et al: Replication of Genetic Polymorphisms Reported to Be Associated with Taxane-Related Sensory Neuropathy in Patients with Early Breast Cancer Treated with Paclitaxel. Clinical Cancer Research 2014, 20(9):2466-2475.

23. Wheeler HE, Gamazon ER, Wing C, Njiaju UO, Njoku C, Baldwin RM, Owzar K, Jiang C, Watson D, Shterev I et al: Integration of cell line and clinical trial genome-wide analyses supports a polygenic architecture of Paclitaxel-induced sensory peripheral neuropathy. Clinical cancer research : an official journal of the American Association for Cancer Research 2013, 19(2):491-499.

24. Leandro-Garcia LJ, Inglada-Perez L, Pita G, Hjerpe E, Leskela S, Jara C, Mielgo X, Gonzalez-Neira A, Robledo $\mathrm{M}$, Avall-Lundqvist $\mathrm{E}$ et al: Genome-wide association study identifies ephrin type A receptors implicated in paclitaxel induced peripheral sensory neuropathy. Journal of medical genetics 2013 , 50(9):599-605.

25. Baldwin RM, Owzar K, Zembutsu H, Chhibber A, Kubo M, Jiang C, Watson D, Eclov RJ, Mefford J, McLeod HL et al: A genome-wide association study identifies novel loci for paclitaxel-induced sensory peripheral neuropathy in CALGB 40101. Clinical cancer research : an official journal of the American Association for Cancer Research 2012, 18(18):5099-5109.

26. Chen El, Crew KD, Trivedi M, Awad D, Maurer M, Kalinsky K, Koller A, Patel P, Kim Kim J, Hershman DL: Identifying Predictors of Taxane-Induced Peripheral Neuropathy Using Mass Spectrometry-Based Proteomics Technology. PloS one 2015, 10(12):e0145816.

27. Sun Y, Kim JH, Vangipuram K, Hayes DF, Smith EML, Yeomans L, Henry NL, Stringer KA, Hertz DL: Pharmacometabolomics reveals a role for histidine, phenylalanine, and threonine in the development of paclitaxel-induced peripheral neuropathy. Breast cancer research and treatment 2018, 171(3):657666. 
28. Jennaro TS, Fang F, Kidwell KM, Smith EML, Vangipuram K, Burness ML, Griggs JJ, Van Poznak C, Hayes DF, Henry NL et al: Vitamin D deficiency increases severity of paclitaxel-induced peripheral neuropathy. Breast cancer research and treatment 2020, 180(3):707-714.

29. Kramer R, Bielawski J, Kistner-Griffin E, Othman A, Alecu I, Ernst D, Kornhauser D, Hornemann T, Spassieva S: Neurotoxic 1-deoxysphingolipids and paclitaxel-induced peripheral neuropathy. Faseb j 2015, 29(11):4461-4472.

30. Cavaletti G, Cornblath DR, Merkies ISJ, Postma TJ, Rossi E, Frigeni B, Alberti P, Bruna J, Velasco R, Argyriou AA et al: The chemotherapy-induced peripheral neuropathy outcome measures standardization study: from consensus to the first validity and reliability findings. Annals of oncology : official journal of the European Society for Medical Oncology 2013, 24(2):454-462.

31. Mielke S, Sparreboom A, Mross K: Peripheral neuropathy: a persisting challenge in paclitaxel-based regimes. European journal of cancer (Oxford, England : 1990) 2006, 42(1):24-30.

32. Lee JJ, Swain SM: Peripheral neuropathy induced by microtubule-stabilizing agents. Journal of clinical oncology : official journal of the American Society of Clinical Oncology 2006, 24(10):16331642.

33. Seretny M, Currie GL, Sena ES, Ramnarine S, Grant R, MacLeod MR, Colvin LA, Fallon M: Incidence, prevalence, and predictors of chemotherapy-induced peripheral neuropathy: A systematic review and meta-analysis. Pain 2014, 155(12):2461-2470.

34. De luliis F, Taglieri L, Salerno G, Lanza R, Scarpa S: Taxane induced neuropathy in patients affected by breast cancer: Literature review. Crit Rev Oncol Hematol 2015, 96(1):34-45.

35. Carlson K, Ocean AJ: Peripheral neuropathy with microtubule-targeting agents: occurrence and management approach. Clin Breast Cancer 2011, 11(2):73-81.

36. Swain SM, Arezzo JC: Neuropathy associated with microtubule inhibitors: diagnosis, incidence, and management. Clin Adv Hematol Oncol 2008, 6(6):455-467.

37. Argyriou AA, Koltzenburg M, Polychronopoulos P, Papapetropoulos S, Kalofonos HP: Peripheral nerve damage associated with administration of taxanes in patients with cancer. Crit Rev Oncol Hematol 2008, 66(3):218-228.

38. Hikino H, Kawashima M, Yamada T, Ozaki N: Motor dominant neuropathy induced by adjuvant therapy with adriamycin and cyclophosphamide followed by dose-dense paclitaxel in a breast cancer patient. Int J Clin Oncol 2006, 11(4):332-335.

39. Augusto C, Pietro M, Cinzia M, Sergio C, Sara C, Luca G, Scaioli V: Peripheral neuropathy due to paclitaxel: study of the temporal relationships between the therapeutic schedule and the clinical quantitative score (QST) and comparison with neurophysiological findings. $J$ Neurooncol 2008 , 86(1):89-99.

40. Molassiotis A, Cheng HL, Lopez V, Au JSK, Chan A, Bandla A, Leung KT, Li YC, Wong KH, Suen LKP et al: Are we mis-estimating chemotherapy-induced peripheral neuropathy? Analysis of assessment methodologies from a prospective, multinational, longitudinal cohort study of patients receiving neurotoxic chemotherapy. BMC Cancer 2019, 19(1):132. 
41. Smith EML, Banerjee T, Yang JJ, Bridges CM, Alberti P, Sloan JA, Loprinzi C: Psychometric Testing of the European Organisation for Research and Treatment of Cancer Quality of Life QuestionnaireChemotherapy-Induced Peripheral Neuropathy 20-Item Scale Using Pooled Chemotherapy-Induced Peripheral Neuropathy Outcome Measures Standardization and Alliance for Clinical Trials in Oncology A151408 Study Data. Cancer Nurs 2019, 42(3):179-189.

42. Smith EML, Zanville N, Kanzawa-Lee G, Donohoe C, Bridges C, Loprinzi C, Le-Rademacher J, Yang JJ: Rasch model-based testing of the European Organisation for Research and Treatment of Cancer (EORTC) Quality of Life Questionnaire-Chemotherapy-Induced Peripheral Neuropathy (QLQ-CIPN20) using Alliance for Clinical Trials in Oncology (Alliance) A151408 study data. Supportive care in cancer : official journal of the Multinational Association of Supportive Care in Cancer 2019, 27(7):2599-2608.

43. Kieffer JM, Postma TJ, van de Poll-Franse L, Mols F, Heimans JJ, Cavaletti G, Aaronson NK: Evaluation of the psychometric properties of the EORTC chemotherapy-induced peripheral neuropathy questionnaire (QLQ-CIPN20). Qual Life Res 2017, 26(11):2999-3010.

44. Lavoie Smith EM, Barton DL, Qin R, Steen PD, Aaronson NK, Loprinzi CL: Assessing patient-reported peripheral neuropathy: the reliability and validity of the European Organization for Research and Treatment of Cancer QLQ-CIPN20 Questionnaire. Qual Life Res 2013, 22(10):2787-2799.

45. Ekholm E, Rantanen V, Antila K, Salminen E: Paclitaxel changes sympathetic control of blood pressure. European journal of cancer (Oxford, England : 1990) 1997, 33(9):1419-1424.

46. Hertz DL: Concerns regarding use of patient-reported outcomes in biomarker studies of chemotherapy-induced peripheral neuropathy. The pharmacogenomics journal 2019, 19(5):411-416.

47. Nyrop KA, Deal AM, Reeder-Hayes KE, Shachar SS, Reeve BB, Basch E, Choi SK, Lee JT, Wood WA, Anders CK et al: Patient-reported and clinician-reported chemotherapy-induced peripheral neuropathy in patients with early breast cancer: Current clinical practice. Cancer 2019, 125(17):2945-2954.

48. Di Maio M, Basch E, Bryce J, Perrone F: Patient-reported outcomes in the evaluation of toxicity of anticancer treatments. Nat Rev Clin Oncol 2016, 13(5):319-325.

49. Kuroi K, Shimozuma K, Ohashi Y, Hisamatsu K, Masuda N, Takeuchi A, Aranishi T, Morita S, Ohsumi $\mathrm{S}$, Hausheer FH: Prospective assessment of chemotherapy-induced peripheral neuropathy due to weekly paclitaxel in patients with advanced or metastatic breast cancer (CSP-HOR 02 study). Supportive care in cancer : official journal of the Multinational Association of Supportive Care in Cancer 2009, 17(8):1071-1080.

50. Chen C-S, Kim J, Garg N, Guntupalli H, Jagsi R, Griggs JJ, Sabel M, Dorsch MP, Callaghan BC, Hertz DL: Chemotherapy-Induced Peripheral Neuropathy Detection via a Smartphone App: Cross-sectional Pilot Study. JMIR Mhealth Uhealth 2021, 9(7):e27502.

\section{Figures}



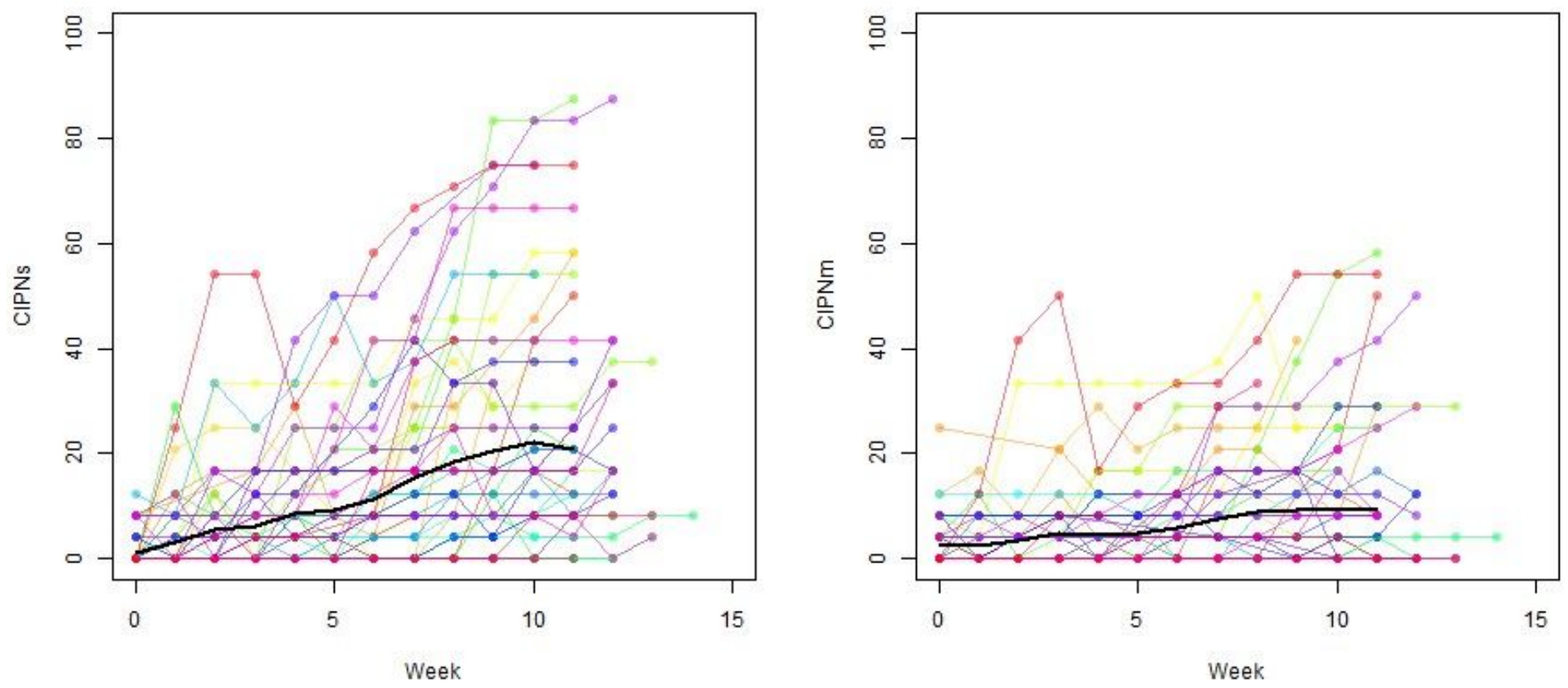

Figure 1

The time course of CIPN $_{S}$ (left) and CIPN $N_{M}$ (right). Each color represents an individual patient that is represented by the same color in both plots. Dots represent CIPN scores at individual time points and are connected by lines linking consecutive weeks. Black solid line indicates the average score at each week. Score values after week 11 were missing in over $80 \%$ of the patients. 


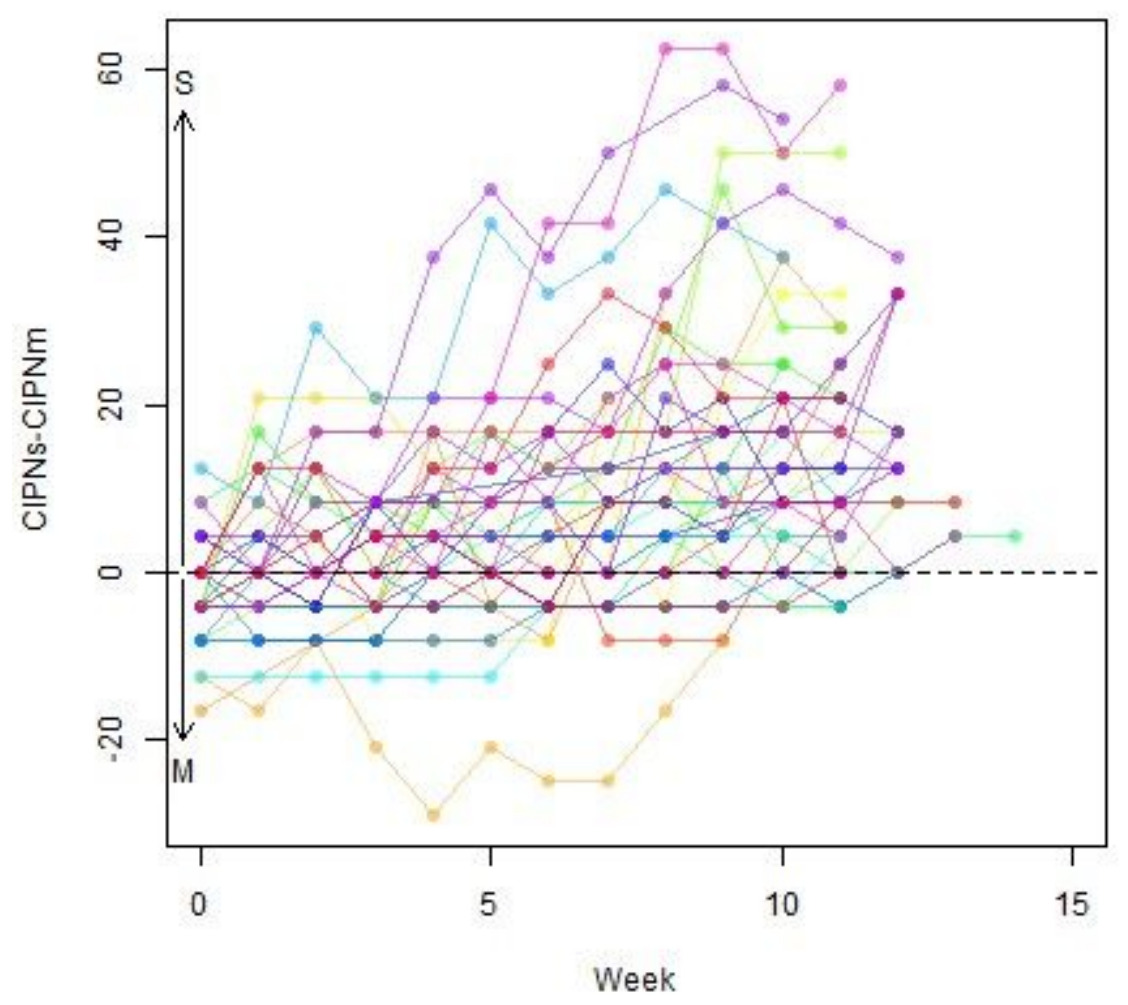

Figure 2

The time course of the difference between sensory and motor CIPN $\left(C_{P} N_{S}-C_{M} N_{M}\right)$. Individual patients were labeled with the same colors as in Figure 1. Dots represent CIPN scores at individual time points and are connected by lines linking consecutive weeks. Values greater than zero indicate sensory-dominant symptoms, and values less than zero indicate motor-dominant symptoms. Most patients had sensorydominant or mixed CIPN symptoms; few patients had motor-dominant symptoms. 


\section{Baseline - Week 11}

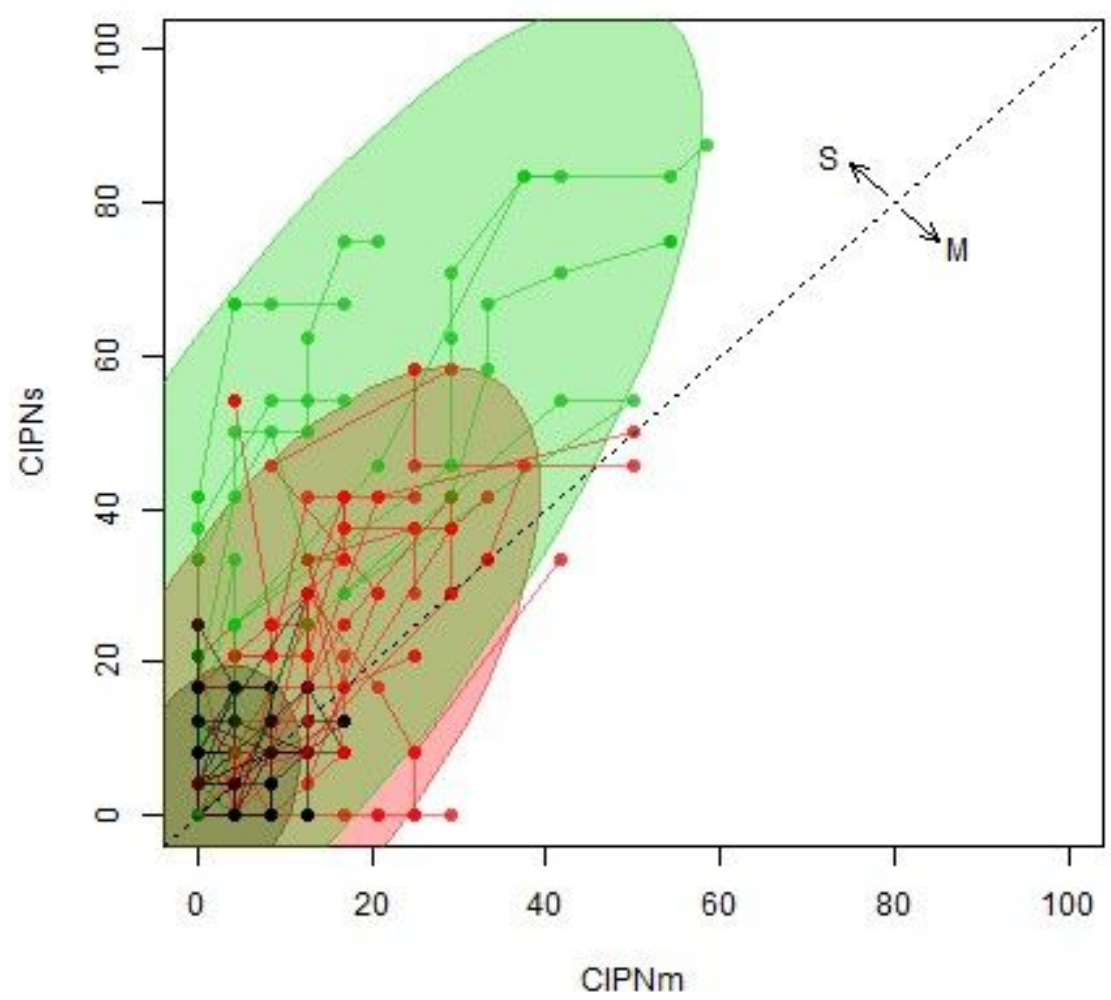

\section{Figure 3}

The scatter plot of CIPN $_{S}$ (sensory CIPN) vs CIPN $_{M}$ (motor CIPN) from baseline to week 11. Values shifting above the diagonal line indicate sensory-dominant symptoms, and values shifting below the diagonal line indicate motor-dominant symptoms. Clusters No CIPN (black), Mixed CIPN (red), and Sensory-dominant Mixed CIPN (green) were identified by k-means clustering. Each line represents a patient. The ellipses indicate $95 \%$ confidence intervals of the CIPN score distributions in each cluster from baseline to week 11 . 


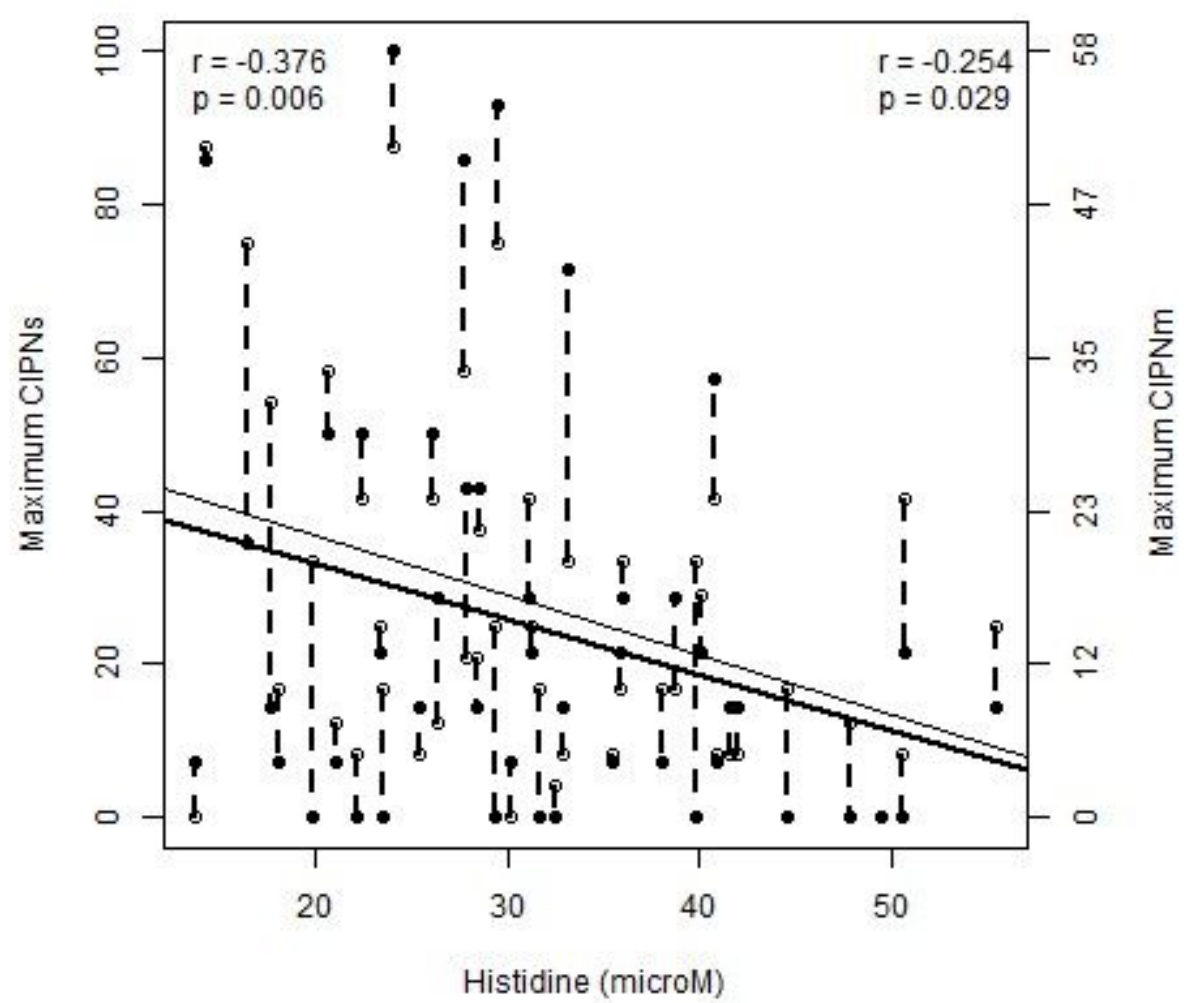

Figure 4

Correlation between histidine levels and maximum CIPN $_{S}$ or CIPN $_{M}$. The scale, correlation coefficient, and p-values of CIPN $_{S}$ are on the left, and the ones of CIPN $_{M}$ are on the right. White dots and thin regression line are CIPN . Black dots and bold regression line are CIPN $_{M}$. Each patient's CIPN $_{S}$ and CIPN $_{M}$ are connected by a dashed line. In general, the CIPN $_{S}$ and CIPN $_{M}$ scores are similar for most patients, resulting in a similar association for histidine with each CIPN subtype.

\section{Supplementary Files}

This is a list of supplementary files associated with this preprint. Click to download.

- SupplementaryMaterial.doc 